Pacific Journal of Mathematics

THE HOMOTOPY TYPE OF THE SPACE OF MAPS OF A
HOMOLOGY 3-SPHERE INTO THE 2-SPHERE

V. L. (VAGN LundsGaARD) 


\title{
THE HOMOTOPY TYPE OF THE SPACE OF MAPS OF A HOMOLOGY 3-SPHERE INTO THE 2-SPHERE
}

\author{
VAGN LUNDSGAARD HANSEN
}

\begin{abstract}
It is proved that if $K$ is a compact, connected polyhedron such that $H^{2}(K ; Z)=0$, then all the components in the space of maps of $K$ into the 2-sphere are homeomorphic. For $K$ a polyhedral homology 3-sphere the common homotopy type of the components is identified and shown to be independent of $K$.
\end{abstract}

1. Introduction and statements of results. Let $K$ and $X$ be a pair of compact, connected polyhedra and let $M(K, X)$ denote the space of (continuous) maps of $K$ into $X$. All mapping spaces will be equipped with the compact-open topology. Corresponding to each homotopy class of maps of $K$ into $X$ there is a (path-) component in $M(K, X)$. For each pair of spaces $K$ and $X$ there arises then a natural classification problem, namely that of dividing the set of components in $M(K, X)$ into homotopy types. The present paper is one in a series of papers, where we search through classical algebraic topology for methods, which are useful in the study of such classification problems.

In [4], information on certain Whitehead products was used to tackle the classification problem for the set of components in the space of maps of the $m$-sphere $S^{m}$ into the $n$-sphere $S^{n}, m \geqq n \geqq 1$, and complete solutions were obtained in the cases $m=n$ and $m=$ $n+1$. If the domain in the mapping space is not a suspension, the problem becomes more delicate, since normally, it is then difficult to construct nontrivial maps between the various components. For a mapping space with a manifold as domain it is sometimes possible to solve the classification problem for the components using information about a corresponding mapping space with a sphere as domain. As an example, knowledge of the fundamental group of the various components in $M\left(S^{2}, S^{2}\right)$ was used in [5] to solve the classification problem for the countable number of components in the space of maps of an orientable closed surface into $S^{2}$. In this paper, we shall investigate spaces of maps into the base space of a principal bundle. We will concentrate mainly on spaces of maps into $S^{2}$, making use of the fact, that $S^{2}$ is the base space in a principal $S^{1}$-bundle, namely the classical Hopf fibration $p: S^{3} \rightarrow S^{2}$.

The main result in this paper is the following 
THEOREM 1. Let $K$ be a compact, connected polyhedron and suppose that the integral cohomology group $H^{2}(K ; Z)=0$. Then all the components in $M\left(K, S^{2}\right)$ are homeomorphic.

Theorem 1 generalizes substantially that part of ([4], Theorem 5.2), which states, that the countably many componts in $M\left(S^{3}, S^{2}\right)$ all have the same homotopy type.

In case $K$ is a polyhedral homology 3-sphere (i.e., $K$ is 3-dimensional and has the same integral homology as $S^{3}$ ) we can identify the common homotopy type of the components in $M\left(K, S^{2}\right)$ and show, that it is independent of $K$. Let $M_{0}\left(K, S^{2}\right)$, respectively $M_{0}\left(S^{3}, S^{2}\right)$, denote that component in $M\left(K, S^{2}\right)$, respectively $M\left(S^{3}, S^{2}\right)$, which consists of the homotopically trivial maps. Then we shall prove

THeOREM 2. Suppose that $K$ is a polyhedral homology 3-sphere. Then the space of maps $M\left(K, S^{2}\right)$ has a countable number of components all of which have the same homotopy type as the component of homotopically trivial maps $M_{0}\left(S^{3}, S^{2}\right)$.

In the proof of Theorem 2 we show that a based map $q: K \rightarrow S^{3}$, which induces an isomorphism between the 3-dimensional homology groups, will induce a homotopy equivalence between $M_{0}\left(K, S^{2}\right)$ and $M_{0}\left(S^{3}, S^{2}\right)$.

Motivated by the spectral sequence constructed by Federer [2], it is natural to raise the question, whether the homotopy of a mapping space is determined just by the cohomology of the domain and the homotopy of the target, at least in favorable cases. Theorem 2 answers this question in the affirmative in a particular case. A generalized version of Theorem 2 appears in Remark 3 below. It would be interesting to know more examples of this kind.

Finally, the author would like to thank the referee for some very valuable constructive [remarks, which helped to improve the presentation of the paper.

2. Spaces of maps into a principal bundle. Throughout $K$ denotes a compact, connected polyhedron. All topological spaces shall have the homotopy type of $C W$-complexes. According to Milnor [7] any mapping space with $K$ as domain will then also have the homotopy type of a $C W$-complex. For any space $X$ and any map $f: K \rightarrow X$, we denote by $M(K, X ; f)$ that component in $M(K, X)$, which contains $f$, i.e., the space of maps of $K$ into $X$ freely homotopic to $f$. When necessary, a space will be equipped with a base point, and for any pair of based spaces $A$ and $B$, we denote by $\pi(A, B)$ the set of based 
homotopy classes of based maps of $A$ into $B$. For $A=S^{n}$, the $n$ sphere, we use the standard notation $\pi_{n}(B)=\pi\left(S^{n}, B\right)$.

For a topological group $G$, the space of maps $M(K, G)$ is a topological group under pointwise multiplication. Similarly, for any right action of $G$ on a space $E$, there is an induced right action of $M(K, G)$ on $M(K, E)$.

Consider now a principal $G$-bundle $p: E \rightarrow B$. By definition $G$ is then a topological group acting freely and properly on $E$, and $p$ is a locally trivial fibration, which identifies $B$ with the orbit space for the action of $G$ on $E$. Clearly the induced action of $M(K, G)$ on $M(K, E)$ is free. For any map $f: K \rightarrow E$ we denote by $\bar{f}=p \circ f: K \rightarrow B$ the composition of $f$ and $p$. Composition with $p$ induces a continuous map $p_{*}: M(K, E) \rightarrow M(K, B)$. It is easy to prove that $p_{*}$ is a Hurewicz fibration over its image, and since $M(K, B)$ has the homotopy type of a $C W$-complex, and therefore is weakly locally contractible, it follows by Fadell ([1], Proposition 4) that $p_{*}$ is an open map. This is used in the proof of the following

Proposition. Let $p: E \rightarrow B$ be a principal G-bundle. Suppose that there is an $M(K, G)$-invariant homeomorphism $\varphi: M\left(K, E ; f_{1}\right) \rightarrow$ $M\left(K, E ; f_{2}\right)$ between the components in $M(K, E)$ defined by the maps $f_{1}, f_{2} \in M(K, E)$. Then $\varphi$ induces a homeomorphism $\bar{\varphi}: M\left(K, B ; \bar{f}_{1}\right) \rightarrow$ $M\left(K, B ; \bar{f}_{2}\right)$.

Proof. A map $\varphi: M\left(K, E ; f_{1}\right) \rightarrow M\left(K, E ; f_{2}\right)$ is called $M(K, G)$ invariant, if for any $f \in M\left(K, E ; f_{1}\right)$ and any $g \in M(K, G)$ such that $f \cdot g \in M\left(K, E ; f_{1}\right)$, the $\operatorname{map} \varphi(f) \cdot g \in M\left(K, E ; f_{2}\right)$ and $\varphi(f \cdot g)=\varphi(f) \cdot g$. Clearly an $M(K, G)$-invariant map $\varphi$ induces a map $\bar{\varphi}$ making the following diagram commutative

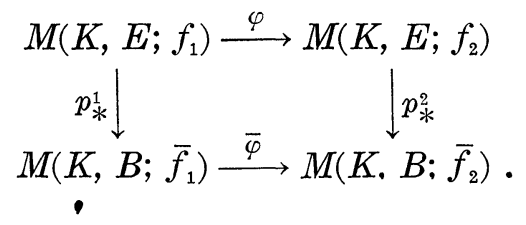

In this diagram, $p_{*}^{1}$ and $p_{*}^{2}$ are restrictions of $p_{*}$. Since the diagram is commutative and $p_{*}^{1}$ is open and surjective, it follows, that $\bar{\phi}$ is continuous if $\varphi$ is continuous.

A homeomorphism $\varphi: M\left(K, E ; f_{1}\right) \rightarrow M\left(K, E ; f_{2}\right)$ is called an $M(K, G)$ invariant homeomorphism if both $\varphi$ and the inverse map to $\varphi$ are $M(K, G)$-invariant maps in the above sense. It is then clear, that the inverse map to an $M(K, G)$-invariant homeomorphisms $\varphi$ induces an inverse map to $\bar{\varphi}$. This proves the proposition. 
3. Proof of Theorem 1. Consider $S^{3}$ as a topological group, with $S^{1}$ as a subgroup, via the natural identification with the topological group of unit quaternions. The action of $S^{1}$ on the right of $S^{3}$ by multiplication defines a principal $S^{1}$-bundle $p: S^{3} \rightarrow S^{2}$ equivalent to the classical Hopf fibration. As in $\S 2$ we get an induced structure as a topological group on $M\left(K, S^{3}\right)$. For two components in $M\left(K, S^{3}\right)$ corresponding to maps $f_{1}, f_{2} \in M\left(K, S^{3}\right)$ we define

$$
\varphi: M\left(K, S^{3} ; f_{1}\right) \longrightarrow M\left(K, S^{3} ; f_{2}\right)
$$

by $\varphi(f)=f_{2} \cdot f_{1}^{-1} \cdot f$. All operations are defined pointwise using the group structure on $S^{3}$. Since $M\left(K, S^{1}\right)$ acts on the right of $M\left(K, S^{3}\right)$, it is obvious that $\varphi$ is an $M\left(K, S^{1}\right)$-invariant homeomorphism. By assumption $H^{2}(K ; Z)=0$, and since the only possible obstruction for lifting a map $\bar{f}: K \rightarrow S^{2}$ to a map $f: K \rightarrow S^{3}$ lies in $H^{2}(K ; Z)$, see Steenrod ([9], Theorem 34.2), it follows, that any component in $M\left(K, S^{2}\right)$ lifts to a component in $M\left(K, S^{3}\right)$. By the proposition in $\S 2$ it follows now immediately that all the components in $M\left(K, S^{2}\right)$ are homeomorphic. This proves Theorem 1.

REMARK 1. Theorem 1 can be generalized as follows. Let $G$ be a Lie group and let $H$ be a closed subgroup of $G$. Then $p: G \rightarrow G / H$ is a smooth principal $H$-bundle. Proceeding exactly as in the proof of Theorem 1 we can prove the

THeOREM. All the components in $M(K, G / H)$, which are images under $p_{*}$ of components in $M(K, G)$, are homeomorphic.

4. Spaces of maps of a polyhedral homology 3-sphere into $S^{2}$. Throughout this section $K$ denotes a polyhedral homology 3sphere, i.e., $K$ is a compact, connected 3-dimensional polyhedron with $H_{1}(K ; \boldsymbol{Z})=H_{2}(K ; \boldsymbol{Z})=0$ and $H_{3}(K ; \boldsymbol{Z}) \cong \boldsymbol{Z}$. By the universal coefficient theorem for cohomology we get then equivalently $H^{1}(K ; Z)=$ $H^{2}(K ; \boldsymbol{Z})=0$ and $H^{3}(K ; \boldsymbol{Z}) \cong \boldsymbol{Z}$. Using elementary obstruction theory for the first isomorphism and the Hopf classification theorem, see Spanier ([8], Corollary 16, p. 431), for the second isomorphism we get

$$
\pi\left(K, S^{2}\right) \cong \pi\left(K, S^{3}\right) \cong H^{3}(K ; Z) \cong Z \text {. }
$$

Thus $M\left(K, S^{2}\right)$ has a countable number of components.

Proof of Theorem 2. Due to Theorem 1, it suffices to consider the component of homotopically trivial maps $M_{0}\left(K, S^{2}\right)$. Evaluation at the base point of $K$ defines a Hurewicz fibration $p^{K}: M_{0}\left(K, S^{2}\right) \rightarrow S^{2}$, the fiber of which is the space of based maps of $K$ into $S^{2}$ homotopic 
to the constant based map, denoted $F_{0}\left(K, S^{2}\right)$. See Spanier ([8], Theorem 2, p. 97 and Corollary 2, p. 400). Similarly, we have the Hurewicz fibration $p^{s}: M_{0}\left(S^{3}, S^{2}\right) \rightarrow S^{2}$ with fiber $F_{0}\left(S^{3}, S^{2}\right)$.

Choose now a base point preserving map $q: K \rightarrow S^{3}$, which induces an isomorphism $q_{*}: H_{3}(K ; \boldsymbol{Z}) \rightarrow H_{3}\left(S^{3} ; \boldsymbol{Z}\right)$. Composition with $q$ induces a map between fibrations

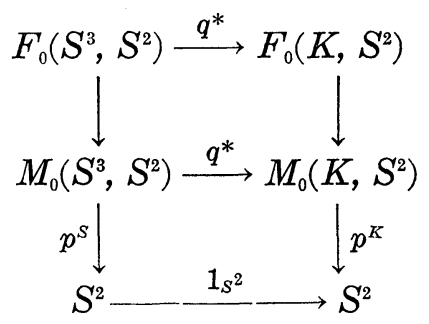

If we take the constant based map as base point in all the mapping spaces involved, then we get for each $i \geqq 1$ a commutative diagram

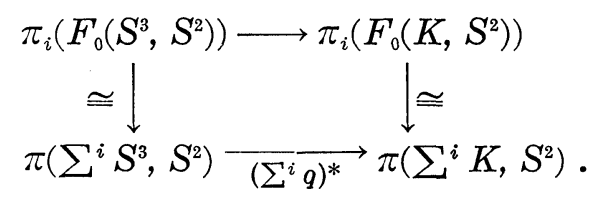

In this diagram $\sum^{i}$ denotes the $i$-fold reduced suspension functor. The vertical maps are natural identifications. The horizontal maps are both induced by $q$.

Consider the map $\sum^{i} q: \sum^{i} K \rightarrow \sum^{i} S^{3}$. From the suspension isomorphism theorem in homology it follows that $\sum^{i} q$ induces an isomorphism between homology groups in all dimensions. Since both $\sum^{i} K$ and $\sum^{i} S^{3}$ for $i \geqq 1$ are simply connected, see Spanier ([8], Corollary 3, p. 454), it follows by a theorem of J. H. C. Whitehead, see Spanier ([8], Corollary 24, p. 405 in connection with Theorem 25 , p. 406), that $\Sigma^{i} q$ is a homotopy equivalence. Hence

$$
\left(\Sigma^{i} q\right)^{*}: \pi\left(\sum^{i} S^{3}, S^{2}\right) \longrightarrow \pi\left(\sum^{i} K, S^{2}\right)
$$

is an isomorphism for all $i \geqq 1$.

In the above map between fibrations, the map between fibers induces therefore an isomorphism between homotopy groups in all dimensions. Using the 5-lemma on the induced map between homotopy sequences for the two fibrations, it follows that $q^{*}: M_{0}\left(S^{3}, S^{2}\right) \rightarrow$ $M_{0}\left(K, S^{2}\right)$ induces an isomorphism between homotopy groups in all dimensions. Hence by a theorem of J.H. C. Whitehead, see Spanier ([8], Corollary 24, p. 405), $q^{*}$ is a homotopy equivalence, and therefore $M_{0}\left(K, S^{2}\right)$ and $M_{0}\left(S^{3}, S^{2}\right)$ have the same homotopy type. 
As already remarked this finishes the proof of Theorem 2.

Corollary. Let $K$ be a polyhedral homology 3-sphere. For an arbitrary map $f: K \rightarrow S^{2}$ we have then

$$
\pi_{i}\left(M\left(K, S^{2}\right), f\right) \cong \pi_{i}\left(S^{2}\right) \oplus \pi_{i+3}\left(S^{2}\right)
$$

for all $i \geqq 1$.

Proof. By Theorem 2 it suffices to consider $M_{0}\left(S^{3}, S^{2}\right)$ with the constant map as base point. Observe now that the fibration $p^{s}$ : $M_{0}\left(S^{3}, S^{2}\right) \rightarrow S^{2}$ with fibre $F_{0}\left(S^{3}, S^{2}\right)$ has a section, namely the section $s: S^{2} \rightarrow M_{0}\left(S^{3}, S^{2}\right)$ of constant maps. Hence the homotopy sequence for $p^{S}$ splits and we get

$$
\begin{aligned}
\pi_{i}\left(M_{0}\left(S^{3}, S^{2}\right)\right) & \cong \pi_{i}\left(S^{2}\right) \oplus \pi_{i}\left(F_{0}\left(S^{3}, S^{2}\right)\right) \\
& \cong \pi_{i}\left(S^{2}\right) \oplus \pi_{i+3}\left(S^{2}\right) .
\end{aligned}
$$

REMARK 2. By appealing to results from infinite dimensional topology we can substitute homotopy type by homeomorphism type in Theorem 2. This follows, since Geoghegan [3] has shown that almost all mapping spaces, and certainly the ones considered here, have the structure of infinite dimensional, separable Hilbert manifolds, and Henderson [6] has shown that two such manifolds are homotopy equivalent if and only if they are homeomorphic.

REMARK 3. As pointed out by the referee, Theorem 2 can be generalized as follows.

Let $K$ and $K^{\prime}$ be compact, connected polyhedra and suppose that there exists a map $q: K \rightarrow K^{\prime}$, which induces an isomorphism $q_{*}$ : $H_{i}(K ; \boldsymbol{Z}) \rightarrow H_{i}\left(K^{\prime} ; \boldsymbol{Z}\right)$ between homology groups in all dimensions $i \geqq 0$. Let also $X$ be an arbitrary connected space. Using exactly the same procedure as in the proof of Theorem 2, we can then prove, that $q$ induces a homotopy equivalence $q^{*}: M_{0}\left(K^{\prime}, X\right) \rightarrow M_{0}(K, X)$. For $X=S^{2}$ and if $H^{2}(K ; \boldsymbol{Z})=H^{2}\left(K^{\prime} ; \boldsymbol{Z}\right)=0$, we get then immediately a generalized version of Theorem 2 .

\section{REFERENCES}

1. E. Fadell, On fiber spaces, Trans. Amer. Math. Soc., 90 (1959), 1-14.

2. H. Federer, A study of function spaces by spectral sequences, Trans. Amer. Math. Soc., 82 (1956), 340-367.

3. R. Geoghegan, On spaces of homeomorphisms, embeddings and functions-I, Topology, 11 (1972), 159-177.

4. V. L. Hansen, The homotopy problem for the components in the space of maps on the n-sphere, Quart. J. Math. Oxford Ser. (2), 25 (1974), 313-321.

5 . - On the space of maps of a closed surface into the 2-sphere, Math. Scand., 
35 (1974), 149-158.

6. D. W. Henderson, Stable classification of infinite dimensional manifolds by homotopy type, Invent. Math., 12 (1971), 48-56.

7. J. Milnor, On spaces having the homotopy type of a $C W$-complex, Trans. Amer. Math. Soc., 90 (1959), 272-280.

8. E. H. Spanier, Algebraic Topology, McGraw-Hill, New York-London, 1966.

9. N. E. Steenrod, The Topology of Fibre Bundles, Princeton Mathematical Series, Princeton University Press, Princeton, N. J., 1951.

Received January 19, 1977 and in revised form October 14, 1977.

MATEMATISK Institut

KobenhavNS UnIVERSITET

Kobenhavn, Denmark 



\section{PACIFIC JOURNAL OF MATHEMATICS}

\section{EDITORS}

RICHARD ARENS (Managing Editor)

University of California

Los Angeles, California 90024

C. W. CURTIS

University of Oregon

Eugene, OR 97403

C. C. MOORE

University of California

Berkeley, CA 94720

\section{J. DUGUNDJI}

Department of Mathematics University of Southern California Los Angeles, California 90007

R. Finn aNd J. Milgram Stanford University Stanford, California 94305

\section{ASSOCIATE EDITORS}

E. F. BeCKenbaCH

B. H. NeumanN

F. WOLF

K. YosHIDA

\section{SUPPORTING INSTITUTIONS}

UNIVERSITY OF BRITISH COLUMBIA CALIFORNIA INSTITUTE OF TECHNOLOGY UNIVERSITY OF CALIFORNIA MONTANA STATE UNIVERSITY UNIVERSITY OF NEVADA, RENO NEW MEXICO STATE UNIVERSITY OREGON STATE UNIVERSITY UNIVERSITY OF OREGON
UNIVERSITY OF SOUTHERN CALIFORNIA STANFORD UNIVERSITY UNIVERSITY OF HAWAII UNIVERSITY OF TOKYO UNIVERSITY OF UTAH WASHINGTON STATE UNIVERSITY UNIVERSITY OF WASHINGTON 


\section{Pacific Journal of Mathematics}

\section{Vol. 76, No. $1 \quad$ November, 1978}

Ata Nuri Al-Hussaini, Potential operators and equimeasurability ......... 1

Tim Anderson and Erwin Kleinfeld, Semisimple nil algebras of type $\delta . \ldots .99$

Stephen LaVern Campbell, Linear operators for which $T^{*} T$ and $T+T^{*}$

commute. III ......................................

Robert Jay Daverman, Special approximations to embeddings of codimension one spheres...............................

Donald M. Davis, Connective coverings of $\mathrm{BO}$ and immersions of projective

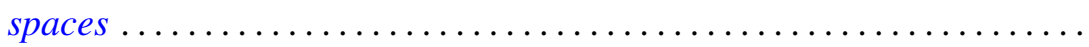

V. L. (Vagn Lundsgaard) Hansen, The homotopy type of the space of maps of

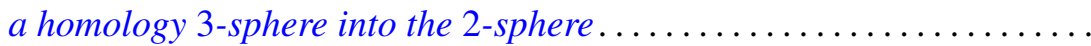

James Victor Herod, A product integral representation for the generalized

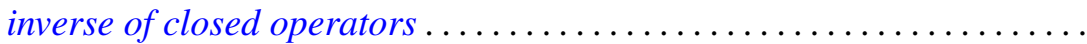

A. A. Iskander, Definability in the lattice of ring varieties ..............

Russell Allan Johnson, Existence of a strong lifting commuting with a

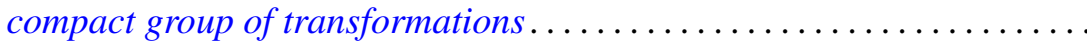

Heikki J. K. Junnila, Neighbornets...................... 83

Klaus Kalb, On the expansion in joint generalized eigenvectors . ......... 109

F. J. Martinelli, Construction of generalized normal numbers . . . . . . . . . 117

Edward O'Neill, On Massey products ....................... 123

Vern Ival Paulsen, Continuous canonical forms for matrices under unitary

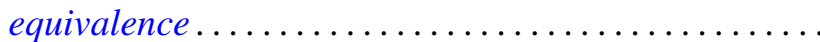

Justin Peters and Terje Sund, Automorphisms of locally compact groups . . . 143

Duane Randall, Tangent frame fields on spin manifolds . . . .

Jeffrey Brian Remmel, Realizing partial orderings by classes of co-simple sets . . . .

J. Hyam Rubinstein, One-sided Heegaard splittings of 3-manifolds ...

Donald Charles Rung, Meier type theorems for general boundary approach

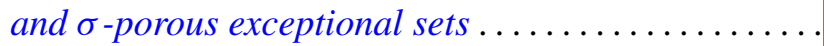

Ryōtarō Satō, Positive operators and the ergodic theorem

Ira H. Shavel, A class of algebraic surfaces of general type constructed from

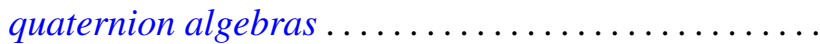

Patrick F. Smith, Decomposing modules into projectives and injectives ....

Sergio Eduardo Zarantonello, The sheaf of outer functions in the polydisc... 